\title{
Performance Analysis by Improving Bit Error Rate (BER) through various Diversity Techniques in Wireless Communication
}

\author{
Joydev Ghosh \\ Dept. of Electronics \& \\ Telecomm. Engg. \\ National Institute of \\ Technology, Durgapur \\ West Bengal, India
}

\author{
Abhishek Dey \\ Dept. of Electronics \& comm. \\ Engg. \\ Bengal College of Engg. \& \\ Tech., Durgapur \\ West Bengal, India
}

\author{
Subham Bachhar \\ Dept. of Electronics \& comm. \\ Engg. \\ Dr. B C Roy Engg. College, \\ Durgapur \\ West Bengal, India
}

\begin{abstract}
In wireless communication systems antenna diversity is an important technique to combat deep fading to improve the system performance and to increase the channel capacity. The fast and random fluctuation of the received signal strength is usually called fading. Diversity technique has been taken into consideration to mitigate the effects of fading by generating several copies of the signal, which experience independent or estimated independent fading, to decrease the probability of instantaneous deep fades. This paper presents the performance analysis of a system in various diversity mechanism environments by improving bit error rate (BER).
\end{abstract}

\section{General Terms}

Diversity Techniques

\section{Keywords}

BPSK modulation, Bit Error Rate(BER), Rayleigh Channel,MRC,Selective Combining(SC), Equal Gain Combining (EGC),Beamforming Technique, Alamouti STBC Technique

\section{INTRODUCTION}

Radio channels are subject to reflection, refraction and diffraction, etc caused by the surrounding obstacles or objects. In this scenario, multipath radio waves (there is consideration of time delay and phase aspect) can interfere with each other constructively or destructively due to multipath propagation exists, resulting in fast and random fluctuation of the received signal strength. The received signal strength fluctuates very fast and this kind of random fluctuation is usually called fading. Diversity technique [1] is invoked to reduce the effects of fading by generating several copies of the signal, which experience independent or estimated independent fading, to reduce the probability of simultaneous deep fades. Space diversity is one of the diversity techniques which use multiple antennas to improve the quality of the radio channels. In space diversity technique, many combining schemes are used to extract the desired signal, such as, Maximum Ratio Combining (MRC),Equal Gain Combining (EGC) and Selection Combining (SC) [2][3]. Among all of them, MRC yields the highest signal to noise ratio (SNR), thus it is the optimum combiner, but also, it is the most complicated one [4].When investigating the performance analysis of diversity schemes, the consideration normally taken is that the received signals at diversity branches are independent and identically distributed (i.i.d) [5]. However, in real-life scenario, an ideal i.i.d fading can rarely be obtained due to the nature of propagation environments and/or inadequate separation among the antennas. For example, when there is a dominant Lineof-Sight (LOS) signal in the propagation, the received signal by the receiver will be rice distributed and then the received signals at diversity branches will not be independent, but correlated with one another. In this condition, the maximum theoretical diversity gain cannot be obtained, so that it is essential to consider the impact of correlation. The impact of correlation on the efficiency of diversity systems has been viewed extensive attention over the past several decades [6][7]. Few correlation models have been proposed in [8][9] and they have been accepted by many researchers to investigate the performance of diversity systems[3][4].

\section{MAXIMAL RATIO COMBINING (MRC) DIVERSITY}

In this approach, the branch of each signal is multiplied by a weight factor which changes in accordance with the signal amplitude. It signifies that branches with strong signal are further boost up, while weak signals are attenuated. In wireless system, maximal-ratio combining is a scheme of diversity combining in which the signals from each channel are summed and the channel gain of each branch is made proportional to the RMS value of signal and inversely proportional to the mean square noise level of that branch(channel). For each channel, there is Different proportionality constant taken into consideration to evaluate the problem. In MRC approach, the signals from all of the channels/branches are weighted according to their individual SNRs and then added together. Hence, the individual signals need to be considered into phase alignment before adding together. In the case of MRC scheme, the ultimate received signals are

$$
\begin{aligned}
& r_{1}=h_{1} s_{o}+n_{1} \\
& r_{2}=h_{2} s_{o}+n_{2}
\end{aligned}
$$

The combined signal is,

$$
\tilde{s}_{0}=h_{1} * r_{1}+h_{2} * r_{2}
$$

Where the fading channel coefficients from antennas 1 and 2 are defined by $h_{1}$ and $h_{2}$, respectively, at time t. $n 1$ and $\mathrm{n} 2$ are independent complex variables with zero mean and unit variance, denoting AWGN samples at 
time t.

$$
\mathbf{Y}=\mathbf{H} * \mathbf{X}+\mathbf{n}
$$

Where the transmitted signals $\mathrm{x}$ is given by

$$
\begin{aligned}
X & =\left[\begin{array}{llllll}
X_{1} & \ldots & \ldots & \ldots & \ldots & X_{k}
\end{array}\right]^{T} \\
& =C\left[\begin{array}{llllll}
V_{1} & \ldots & \ldots & \ldots & \ldots & V_{k}
\end{array}\right]^{T}
\end{aligned}
$$

A scheme is assumed, which consists of $\mathrm{K}$ antennas for transmission and $\mathrm{L}$ antennas for reception. The channel contains of $\mathrm{K}^{*} \mathrm{~L}$ statistically independent channel coefficients. It can be more precisely represented by a matrix

$$
H=\left[\begin{array}{ccc}
H_{11} & \ldots & H_{1 k} \\
\vdots & \ddots & \vdots \\
H_{L 1} & \ldots & H_{L k}
\end{array}\right]
$$

The entries of $\mathrm{H}$ are modeled as independent and identically distributed (i.i.d.) complex gaussian random variables with zero mean and variance 0.5 per dimension and the elements of $\mathrm{n}$ are modeled as i.i.d. complex Gaussian random variables with zero mean and variance $N o / 2$ per dimension. The utmost representative of non line of sight (NLOS) wireless radio channel propagation is the Rayleigh distribution and hence the multiple inputs multiple output (MIMO) channel capacity has been taken into measure for Rayleigh fading channel model includes the idea of MRC scheme. It is suppose to be assumed that the receiver is known the channel state information (CSI) and there is no as such CSI known at the transmitter, and the channel fading coefficients remain unchanged over the entire frame and changes from one frame to another. The theoretical information capacity of such full complex multiple inputs multiple output (MIMO) systems which use all available transmit and receive antennas is given by

$$
\begin{array}{r}
C_{\text {full }}=\log _{2} * \operatorname{det}\left(I_{N R}+\frac{E_{S}}{N_{T} N_{0}}\right) \\
\text { If } \mathrm{N}_{\mathrm{R}}<\mathrm{N}_{\mathrm{T}} \ldots \ldots \ldots
\end{array}
$$

Where $\mathrm{HH}$ is component wise transpose conjugate of $\mathrm{H}$. INR is $\mathrm{NR} * \mathrm{NR}$ identity matrix

The noise vector is expressed as

$$
N=\left[n_{1} \ldots \ldots \ldots \ldots n_{L}\right]^{T}
$$

Note that noise is considered to be AWGN and uncorrelated with the signals. The received signals are weighted and added together to produce the estimate of the symbol/pilot. As the wireless radio channel is a time-varying multipath channel and is subject to physical propagation path loss .The time-variations are caused by the medium changes as the the motion of users from cell centre to cell edge or vice-versa. The propagation losses are related to both the atmospheric propagation and the terrain configuration. It has been seen that the multipath aspect is caused by reflection from various objects such as building or trees that surround the mobile unit. The receive signal affect the performance of the receiver due to these propagation phenomena which results in an enhance of bit error rate (BER). The MR channel performance is obtained for different modulation technique which viewed the four major performance types in terms of signal to noise ratio $\left(\mathrm{E}_{\mathrm{b}} / \mathrm{N}_{0}\right)$ versus error probability. Hence, if $h_{i}$ is a Rayleigh distributed random variable, then $h_{22}$ is a chi-squared random variable with two degrees of freedom.
The pdf of $\gamma_{i}$ is

$$
P\left(\gamma_{i}\right)=\frac{1}{\left(E_{b} / N_{0}\right)} e^{-\gamma_{i} /\left(E_{b} / N_{0}\right)}
$$

Since the effective bit energy to noise ratio is the sum of $\mathrm{N}$ such random variables. The pdf is,

$$
P(\gamma)=\frac{1}{(N-1) !\left(E_{b} / N_{0}\right)^{N}} \gamma^{N-1} e^{-\frac{\gamma}{\left(E_{b} / N_{0}\right)}}
$$

If you recall, in the post on BER computation in AWGN, with bit energy to noise ratio of $E_{b} / N_{0}$, the bit error rate for BPSK in AWGN is derived as

$$
P_{b}=\frac{1}{2} \operatorname{erfc}\left(\sqrt{\frac{E_{b}}{N_{0}}}\right)
$$

Given that the effective bit energy to noise ratio with maximal ratio combining (MRC) is, the total bit error rate (BER) which integrals of the conditional BER integrated over all possible values of $\gamma$. This equation is given by

$$
P_{e}=P^{N} \sum_{k=0}^{N-1}(N-1+K)(1-P)^{k}
$$

Where,

$$
\left.P=\frac{1}{2}-\frac{1}{2}\left(1+1 / E_{b}\right)_{N_{0}}\right)^{-0.5}
$$

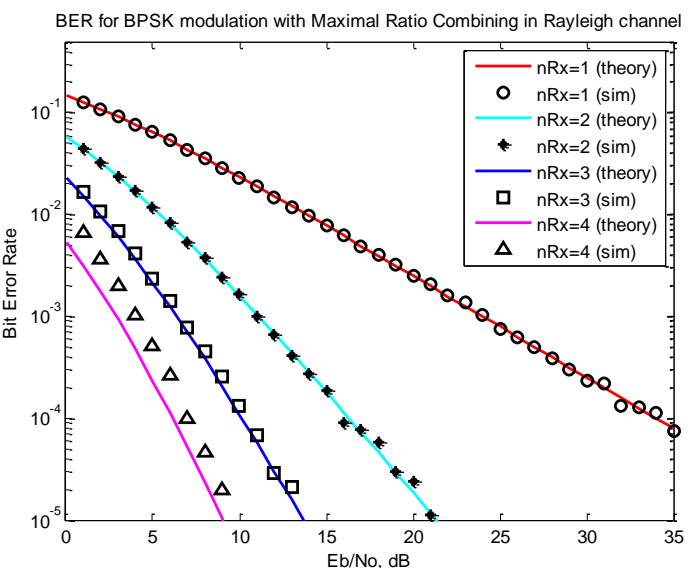

Fig.1: Computation of BER by MRC diversity.

In uncoded systems under rayleigh fading, the central limit theorem can be applied to the total interference, which can therefore be estimated to be gaussian distributed .The average error rates can thus be calculated by remediating the conditional error probability (CEP), i.e., the error rate under AWGN, over the output SINR. Above simulation shows that there is an improvement in BER i.e. BER decreases with the increasing no of receiving antenna. 


\section{EQUAL GAIN COMBINING (EGC) DIVERSITY}

The maximal ratio combining (MRC) technique needs an exact estimate of the channel amplitude gain, which enhances the receiver complexity. An alternative way out is to weight all the signals equally after coherent detection (which eliminates the phase distortion).The coherent detected signals from all the branches are simply added and applied to the decision device. As the receiver does not need estimate the amplitude fading, its complexity is reduced as compared with that of maximal ratio combining (MRC).

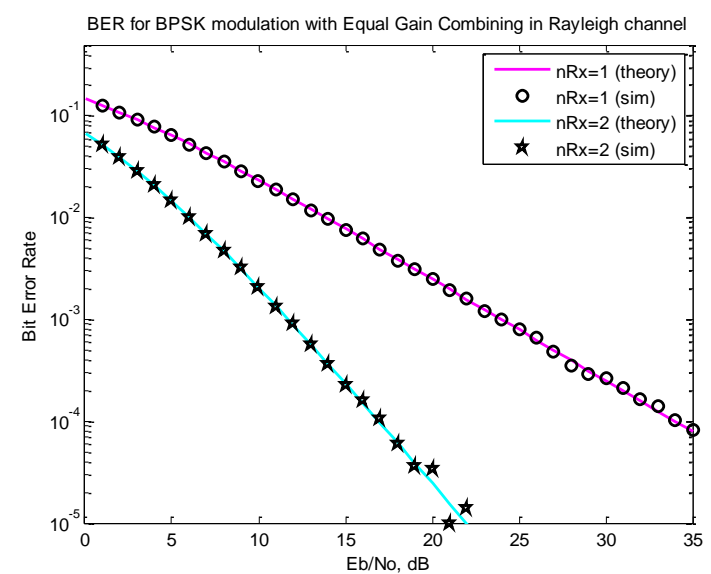

Fig.2: Computation of BER by EGC diversity

\section{SELECTIVE COMBINING (SC) DIVERSITY}

Note that wireless agents (e.g. mobile units or wireless sensors) using higher-order receiver diversity which can not be installed by MRC because of power and processing limitation. In addressing this problem, selective combining diversity is proposed. In addition this scheme provides best trade off between receiver complexity and performance of the system.

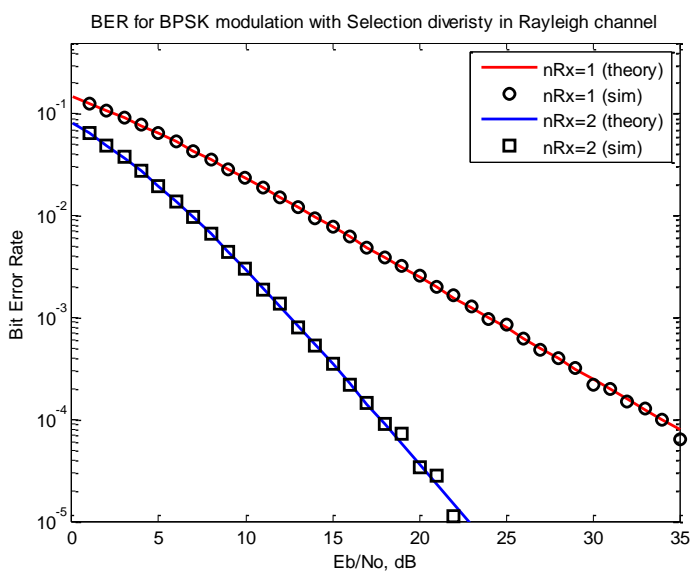

Fig.3: Computation of BER by SC diversity

In this approach, receiver look after the SNR value of each diversity channel and selects/picks up the one with the highest SNR value for signal detection compared with the preceding two techniques. Selective diversity is much easier to implement without much performance degradation, especially for the reverse link transmission where the diversity branches can be physically located in different base stations, which would make it difficult to use maximal ratio combining (MRC) or equal gain combining (EGC).

\section{BEAMFORMING TECHNIQUE}

Beamforming Technique is a signal processing technique used for a myriad of purposes, such as detecting the presence of a signal, estimating the direction of arrival, and boost up a desired signal, competing sources and reverberation. Beamforming has been embraced by the audio research society, mostly to separate or extract speech for noisy environment. Beamforming techniques significantly approach the difficulties from a spatial point of view. A Beamformer is a signal processor used together with an array of microphone to design a spatial filter which extracts a signal from a desire direction and decrease the unsuitable signals from other directions. This is achieved by combining elements in a phased array in such a way that signals at particular angles experience constructive interference while others experience destructive interference. Beamforming can be used at both the transmitting and receiving ends in order to achieve spatial selectivity.

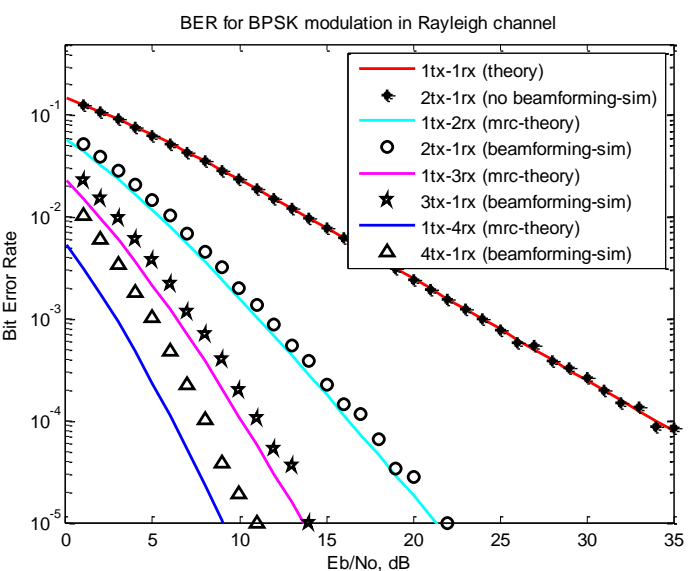

Fig.4: Computation of BER by Beamforming Technique.

In general, beamforming for signals such as voice or speech can be functioned in the time domain. In time domain, a finite impulse response (FIR) filter is applied to each microphone (input transducer) signal, and the filter outputs combined to form the Beamformer output. Beamforming can be carried out by computing multichannel filters whose output is $\tilde{s}(t)$ an estimate of the desired source signal. The output can be expressed as:

$$
\tilde{s}(t)=\sum_{i=1}^{N} \sum_{p=0}^{p-1} w_{i, p} X_{i}(t-p)
$$

\section{ALAMOUTI SPACE TIME BLOCK CODED (STBC) TECHNIQUE}

In fast time varying system, the difficulties of picking up the channel coefficient needs for symbol decoding generally arises .A solution of this specific problem is the Alamouti STBC technique. In this specific case we have considered multiple transmit/multiple receive antenna system with 2 transmit and 2 receive antennas.

The system equation can be written as-

$$
Y(n)=H(n) X(n)+W(n)
$$

where $\mathrm{H}$ is the channel matrix, $\mathrm{y}(\mathrm{n})$ is the $2 \times 1$ receive vector, $\mathrm{x}(\mathrm{n})$ is the $2 \mathrm{x} 1$ transmit vector and, $\mathrm{w}(\mathrm{n})$ is the $2 \mathrm{x} 1$ 
white gaussian noise vector. For an Almouti encoded system, data is encoded in blocks of two and each source block is

$$
\begin{array}{r}
x(2 n)=\left[\begin{array}{l}
x_{1}(2 n) \\
x_{2}(2 n)
\end{array}\right] \\
x(2 n+1)=\left[\begin{array}{c}
-x_{2}^{*}(2 n) \\
x_{1}^{*}(2 n)
\end{array}\right]
\end{array}
$$

Where, '*' denotes complex conjugation. Here we have 2 receivers, the received vector (1) during the time intervals $2 \mathrm{n}$ and $2 \mathrm{n}+1$, can be expressed as

$$
\begin{aligned}
& \tilde{y}(2 n) \triangleq\left[\begin{array}{c}
y(2 n) \\
y(2 n+1)
\end{array}\right] \\
& =\widetilde{H}(2 n) x(2 n)+\widetilde{w}(2 n)
\end{aligned}
$$

Where, $\mathrm{x}(2 \mathrm{n})$ is the $2 \mathrm{X} 1$ transmit vector and $\widetilde{w}(n) \triangleq$ $\left[w^{T}(2 n) w^{H}(2 n+1)\right]$ is the $4 \mathrm{X} 1 \mathrm{H}(2 \mathrm{n})$ is defined as-

$$
\widetilde{H}(2 n) \triangleq\left[\begin{array}{c}
H(2 n) \\
\widetilde{H}^{*}(2 n+1)
\end{array}\right]
$$

While,

$$
\widetilde{H}(2 n+1)=\left[\begin{array}{ll}
h_{12}(2 n+1) & -h_{11}(2 n+1) \\
h_{22}(2 n+1) & -h_{21}(2 n+1)
\end{array}\right]
$$

The idea of symbol aided channel interpolation is to be embedded known as pilots (known as symbol sequences) in each frame, and to estimate the channel responses at these time instances. Using these datas, the channel responses at the other instances are computed by interpolating the available estimates, and it is analogous to the reconstruction of a sampled band limited signal. The length of the frame must be less than the Nyquist rate, i.e.

$$
L_{f} \leq 1 / 2 f_{d} T
$$

So that we can reconstruct the channel response.

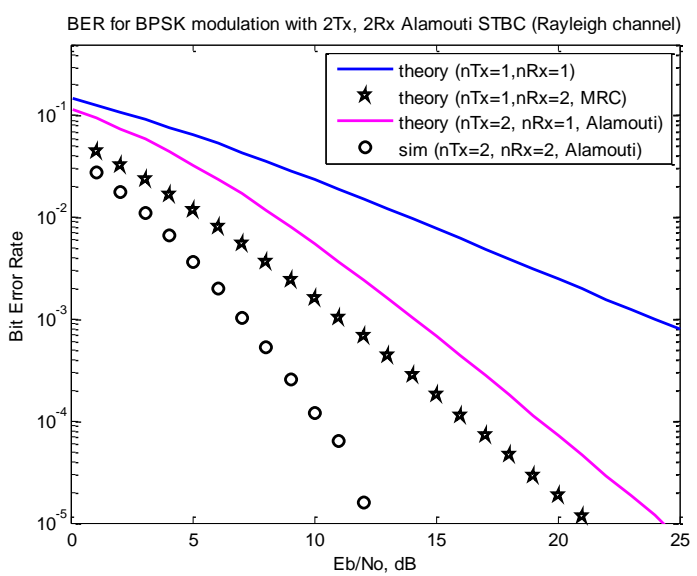

Fig.5: Computation of BER by Alamouti STBC

Where, $T$ is the symbol period and $f_{d}$ is the maximum Doppler frequency of the channel. We suppose to be assumed that the first symbol of each frame is the pilot symbol block. We have considered that the pilot symbol block of the frame to be detected at time zero without any loss of generality at the receiver. By using the pilots, we can therefore obtain estimates of channels at time intervals of $\mathrm{L}_{\mathrm{f}}$. The channel responses of the (i, m)th element of $\mathrm{H}(\mathrm{n}), \mathrm{h}^{\mathrm{i}, \mathrm{m}}$ (1), for the time epochs 1 to $L_{\mathrm{f}}-1$ can then be interpolated by using

$$
\widetilde{H}^{(i, m)}(l)=f^{T}(l) \tilde{h}^{(i, m)}, \mathrm{l}=1, \ldots .,\left(\mathrm{L}_{\mathrm{f}}-1\right)
$$

Where $\mathrm{f}(\mathrm{l})$ is the set of interpolation filter coefficients for the lth symbol in the frame, and $h^{(i, m)}$ is the vector of pilot estimated channel responses defined as

$$
\left[\tilde{h}^{(i, m)}\left(\left\lfloor-\frac{\eta}{2}+1\right\rfloor L_{f}\right) \ldots . \tilde{h}^{(i, m)}\left(\left|\frac{\eta}{2}\right| L_{f}\right)\right]^{T}
$$

The number of adjacent reckons used to aid the interpolation, $\eta$ can be taken base on the system delay that can be tolerated.

\section{CONCLUSIONS}

In this paper, we have proposed an analytical frame work and simulation testbed for performance analysis by improving bit error rate (BER) through various diversity techniques in wireless communication. As per system requirement and situation based condition ,any one diversity cum multiantenna transmission technique can be utilised to mitigate deep fading or multipath fading to improve BER results in better system functioning. Among the MRC,EGC and SC schemes, MRC achieves the best performance difference, followed by EGC. However, with a low diversity order, the performance differences among the three combining techniques are not very significant. An significant application of the Alamouti Space Time Block Coded (STBC) Technique scheme is to facilitate diversity refinement at all the remote units in a wireless system, using two transmit antennas $\left(\mathrm{T}_{\mathrm{x}}\right)$ at the base stations(BS) instead of two receive antennas $\left(R_{x}\right)$ at all the remote terminals. The scheme does not require any feedback from the receiver to the transmitter and its reckoning complexity is quite similar to MRC. While compared with MRC in aspect of remain same radiated power, the Alamouti STBC diversity technique has a 3-dB penalty due to the simultaneous transmission of two different symbols(pilots) from two transmit antennas at the base station (BS).

Due to spectral scarcity, the femtocells and macrocells have to reuse the total allocated frequency band partially or totally which leads to inter cell interference (ICI) results from the motion of users from cell centre to cell edge resulting in power reduction of the signal transmitted from the cell centre while interference signals from neighbour cell is increased.MRC/Alamouti STBC scheme would be absolutely perfect to take the measure by simulated BER of very advanced OFDM wireless system where frequency are reusing due to spectral scarcity and ICI is the main challenging issue.

\section{REFERENCES}

[1] F. Javier L'opez-Mart'inez, Eduardo Martos-Naya, KaiKit Wong, J. Tom'as Entrambasaguas," Closed-Form BER Analysis of Almouti-MRC Systems with ICSI in Ricean Fading Channels", ieee communications letters, vol. 15, no. 1,January 2011.

[2] Siavash M. Alamouti," A Simple Transmit Diversity Technique for Wireless Communications", IEEE journal on select areas in communications, vol. 16, no. 8, October 1998.

[3] Zhuo Chen, Member, IEEE, Jinhong Yuan, Member, IEEE, and Branka Vucetic,Fellow, IEEE," Analysis of Transmit Antenna Selection/Maximal-Ratio Combining 
in Rayleigh Fading Channels"ieee transactions on vehicular technology,vol. 54, no. 4, July 2005

[4] A. Shah, Senior Member, IEEE and A. M.Haimovich," Performance Analysis of Maximal Ratio Combining and Comparison with Optimum Combining for Mobile Radio Communications with Cochannel Interference", ieee transactions on vehicular technology, vol. 49, no. 4, July 2000

[5] M. Ju, H.-K. Song and I.-M. Kim, "Exact BER analysis of distributed Alamouti's code for cooperative diversity networks," IEEE Trans. Commun., vol. 57, no. 8, pp. 2380-2390, Aug. 2009.

[6] F. J. Lopez-Martinez, E. Martos-Naya, J. F. Paris, and U. Fernandez-Plazaola ," Generalized BER analysis of $Q A M$ and its application to MRC under imperfect CSI and interference in Ricean fading Channels"ieee Trans. Veh. Technol., vol. 59, no. 5, pp. 2598-2604, June 2010.

[7] W. Li and N. Beaulieu, "Effects of channel-estimation errors on receiverselection-combining schemes for Alamouti MIMO systems with BPSK,"IEEE Trans.Commun., vol. 54, no. 1, pp. 169-178, Jan.2006.

[8] Yong Soo Cho, Jaekwon Kim, Won Young Yang, Chung G. Kang," mimoofdma wireless communications with matlab".

[9] M. Luo, G. De La Roche, G. Villemaud,J. Gorce, D. Umansky, and J. Zhang,"Simulation of wide band multipath fast fading based on finite difference method," in IEEE Vehicular Technology Conference, no.2011.

[10] J.-M. Gorce, G. Villemaud, and P. Flipo, “On simulating propagation for OFDM/MIMO systems with the MRFDPF model," in the Fourth European Conference on Antennas and Propagation (EuCAP), 2010, pp. 1-5.

[11] J.-M. Gorce , K. Jaffres-Runser, and G. de la Roche, "Deterministic approach for fast simulations of indoor radio wave propagation," IEEE Transactions on Antennas and Propagation, vol. 55, no. 3,pp. 938 $948,2007$.

[12] Suvarna P. Jadhav, Vaibhav S. Hendre," Performance of Maximal Ratio Combining(MRC) MIMO Systems for Rayleigh Fading Channel", International Journal of Scientific and Research Publications, Volume 3, Issue 2, February 20131 ISSN 2250-3153,PP-(1-4).

[13] Meiling Luo, Guillaume Villemaud, Jialai Weng,Jean Marie Gorcey, Jie Zhang," Realistic Prediction of BER and AMC with MRC Diversity for Indoor Wireless Transmissions", Wireless Communications and Networking Conference (WCNC), 2013 IEEE (2013) DOI : 10.1109/WCNC.2013.6555227

[14] Dhruv Malik, Deepak Batra," Comparison of various detection algorithms in a MIMO wireless communication receiver", International Journal of Electronics and Computer Science Engineering, ISSN 2277-1956/V1N31678-1685. 